cytotoxicity of organic solvents (e.g., Dimethyl Sulfoxide (DMSO)). Aqua Embolic System (AES) is a new LEM, which is mainly composed of multiple polysaccharides. AES, when injected via a microcatheter, immediately forms a solid and elastic hydrogel cast upon exposure to $\mathrm{Ca} 2+$ in the bloodstream. The use of organic solvents, e.g., DMSO, is not required. The performance of AES was evaluated using an established AVM model utilizing swine rete-mirabile.

Methods Under general anesthesia, the left ascending pharyngeal artery (APA) of Yorkshire swine $(40 \mathrm{~kg})$ was catheterized using a microcatheter (ID:0.013 inches), and AES was slowly injected into the rete-mirabile under fluoroscopy. The following parameters were assessed to evaluate the embolization performance of the AES; 1) the amount of AES required for the complete occlusion of the feeding artery, 2) injection speed, 3) radiopacity during the deployment, and 4) incidence of catheter entrapment after the injection. The same evaluation was performed on the contralateral rete-mirabile and the left renal artery as well.

Results 12 arteries in 4 swine were treated, and all arteries were completely occluded without technical complications. The injected materials immediately formed AES cast in all vessels, followed by the reflux over the tip of the microcatheter. All catheters were withdrawn without any sign of catheter entrapment. The AES mixed with tantalum-based contrasts medium showed sufficient radiopacity under fluoroscopy. With the injection speed of $0.02 \mathrm{ml} / \mathrm{sec}$, the average volume required was $0.85 \mathrm{~mL}$ for the APA and $2.9 \mathrm{~mL}$ for the renal artery. No increased thrombogenicity or vasospasm near the treated lesion was observed during the procedure.

Conclusions AES, which is a DMSO free, non-adhesive polysaccharides based LEM, may be used as an embolic material for the treatment of hemorrhagic stroke caused by cerebrovascular diseases, such as brain AVM.

A: common carotid artery injection (AP view) shows left ascending pharyngeal artery (APA) and left rete mirabile (red arrow). B: AES is injected from the microcatheter. The AES reflux is reaching the tip of the microcatheter. C: With continuous injection of AES, the microcotheter tip is embedded in the cost of AES. D. Post treatment angiogram shows complete occlusion of the left rete mirabile and APA

Disclosures I. Yuki: 1; C; ICTS Grant, POP Grant. K. Ohkawa: None. S. Li: None. E. Steward: None. B. Nguyen: None. F. Hsu: None. J. Xu: None. S. Suzuki: None.

\section{P-027 ULTRA-EARLY FUNCTIONAL IMPROVEMENT AFTER STROKE THROMBECTOMY - PREDICTORS AND IMPLICATIONS}

${ }^{1} \mathrm{~S}$ Desai", ${ }^{1} \mathrm{D}$ Tonetti, ${ }^{1} \mathrm{G}$ Nayar, ${ }^{1} \mathrm{~A}$ Morrison, ${ }^{1} \mathrm{~K}$ Shah, ${ }^{2} \mathrm{~T}$ Jovin, ${ }^{1} \mathrm{~A}$ Jadhav. ${ }^{1}$ University of Pittsburgh Medical Center, Pittsburgh, PA; ${ }^{2}$ Cooper University, Camden, NJ

\subsection{6/neurintsurg-2021-SNIS.63}

Introduction Neurocritical care is routinely offered to patients post neurothrombectomy of anterior large vessel occlusion (LVO) strokes. We aim to study the relationship between immediate improvement in NIHSS score on outcomes post thrombectomy and potential implications for requiring neurocritical care.

Methods We performed a retrospective review of anterior LVO (internal carotid/proximal middle cerebral artery) patients undergoing neurothrombectomy between January 2015-
December 2018. Demographic, clinical (NIHSS score on admission and within 30 minutes post recanalization, time last know well-TLKW), and imaging (ASPECTS, TICI, intracranial hemorrhage) information was analyzed. Ultra-early functional improvement (Ultra-EFI) was defined as NIHSS score $<6$ within 30 minutes of successful recanalization. We analyzed the incidence and predictors of ultra-EFI and explored reasons for neurological decline post ultra-EFI.

Results Of the 343 anterior LVO patients undergoing neurothrombectomy, mean age was $71 \pm 15$ and $46 \%$ were males. Mean NIHSS was $17 \pm 6$ and TLKW to arrival was $9 \pm 11$ hours. Ultra-EFI was observed in 23\% (79/343) of patients. Independent predictors of ultra-EFI include lower pre-treatment NIHSS (), favorable ASPECTS (), and lower systolic blood pressure (). Rates of 90-day-mRS 0 -2 were higher $(71 \%$ VS $33 \%, \mathrm{P}<0.01)$ and the rate of mortality (8\% VS $28 \%$, $\mathrm{P}<0.01$ ) was lower in the ultra-EFI group compared to the non-ultra-EFI group. Amongst patients with ultra-EFI, 1.3\% $(1 / 79)$ experienced increase in NIHSS by $\geq 4$ points. This patient received thrombolysis, achieved TICI-2B recanalization, and follow-up neuroimaging revealed a parenchymal hemorrhage and an infarct volume of $44 \mathrm{ml}$. None of the patients required continuous antihypertensive infusions.

Conclusion Approximately 23\% of anterior LVO stroke patients undergoing neurothrombectomy have a NIHSS score of $<6$ within 30 minutes of successful recanalization. Approximately $1 \%$ of them experience significant decline in neurological status within 24 hours of the procedure with the majority achieving functional independence at 90 days. Need for advance neurocritical care should be re-evaluated in these patients.

Disclosures S. Desai: None. D. Tonetti: None. G. Nayar: None. A. Morrison: None. K. Shah: None. T. Jovin: None. A. Jadhav: None.

\section{P-028 OPHTHALMIC ARTERY FLOW DIRECTION CHANGES IN INTRA-ARTERIAL TREATMENT OF RETINOBLASTOMA}

${ }^{1} \mathrm{M}$ Feldman*, ${ }^{1} \mathrm{H}$ Grimaudo, ${ }^{1} \mathrm{~S}$ Roth, ${ }^{2} \mathrm{H}$ Vance, ${ }^{3} \mathrm{~A}$ Daniels, ${ }^{1} \mathrm{M}$ Froehler. ${ }^{1}$ Neurosurgery, Vanderbilt University Medical Center, Nashville, TN; ${ }^{2}$ Pediatric Neurosurgery, Vanderbilt University Medical Center, Nashville, TN; ${ }^{3}$ Department of Ophthalmology and Visual Sciences, Vanderbilt University Medical Center, Nashville, TN

\subsection{6/neurintsurg-2021-SNIS.64}

Introduction/Purpose The introduction of catheter-administered intra-arterial chemotherapy (IAC) has changed the treatment paradigm for retinoblastoma (RB), the most common ophthalmologic malignancy in children. The variable nature of ophthalmic artery flow has led to the development of multiple endovascular techniques for drug delivery. These methods are based on the direction of ophthalmic artery filling, either retrograde from anastomoses with branches of the external carotid artery (ECA) or anterograde from the internal carotid artery (ICA). These include direct catheterization of the ophthalmic artery, ECA branch selection, use of an ICA balloon occlusion with proximal IAC administration, and balloon occlusion of the ECA to halt anastamotic flow to the OA.We sought to evaluate both direction of ophthalmic artery flow at the onset of IAC, as well as identify reversal of ophthalmic artery flow during the course of treatment.

Materials and Methods We performed a retrospective analysis of the most recent 15 consecutive patients with $\mathrm{RB}$ who completed treatment with IAC at our tertiary care center. 